\title{
Design, Fabrication, and Test of a Superconducting Dipole Magnet Based on Tilted Solenoids
}

\author{
S. Caspi, D.R. Dietderich, P. Ferracin, N.R. Finney, M.J. Fuery, S.A. Gourlay and A.R. Hafalia
}

\begin{abstract}
It can be shown that, by superposing two solenoid-like thin windings that are oppositely skewed (tilted) with respect to the bore axis, the combined current density on the surface is "costheta" like and the resulting magnetic field in the bore is a pure dipole. As a proof of principle, such a magnet was designed, built and tested as part of a summer undergraduate intern project. The measured field in the $25 \mathrm{~mm}$ bore, 4 single strand layers using NbTi superconductor, exceeded $1 \mathrm{~T}$. The simplicity of this high field quality design, void of typical wedges end-spacers and coil assembly, is especially suitable for insert-coils using High Temperature Superconducting wire as well as for low cost superconducting accelerator magnets for High Energy Physics. Details of the design, construction and test are reported.
\end{abstract}

Index Terms - Superconducting magnet, pure dipole, insert magnet, tilted solenoid.

\section{INTRODUCTION}

$I^{n}$ n a published paper by D.I. Meyer and R. Flasck in 1970 [1] 1 the authors discussed the magnetic field resulting from the superposition of two oppositely skewed (tilted) solenoids with respect to the bore axis (Fig. 1). The resulting superimposed current density cancels the azimuthal component of the magnetic field and enhances its axial component in proportion to $J_{0} \cos \theta$. The idea reappeared in a series of publications [2]-[5] that, by not crediting the 1970's publication, may have been unaware of it. With renewed interest in high field $\mathrm{Nb}_{3} \mathrm{Sn}$ magnets at LBNL and CERN [6], [7] the idea gained interest leading to the construction a small dipole magnet.

As part of LBNL summer student program in 2005, we have designed, built and tested a superconducting dipole that is based on the concept of tilted solenoid windings. The design and analysis was done with the program MathCad $®$ and carried out by two undergraduate students over a period of three weeks. Impressed by the simplicity of the design and low construction cost (in full agreement with all previously published conclusions) we have extended the concept and are

Manuscript received August 27, 2006. This work was supported by the Director, Office of Energy Research, Office of High Energy and Nuclear Physics, High Energy Physics Division, U. S. Department of Energy, under Contract No. DE-AC02-05CH11231.

Authors are with Lawrence Berkeley National Laboratory, Berkeley, CA 94720 USA (e-mail: s_caspi@lbl.gov).

N.R. Finney and MJ. Furry are undergraduate students at UCB an USD. presently building a similar quadrupole magnet. Advantages of this concept are as follows: 1) high field quality in an extended dynamic range, 2) no field optimization required, 3) small number of components - wedges and spacers not needed, 4) coil assembly not needed (all poles are wound together around a single bore), 5) small bore sizes not limiting "cos-theta" windings, 6) continuous windings and ease of magnet "grading". There are also other open issues such as magnet protection and pre-stress that will need to be addressed in the future. The concept can work well for "stand alone" accelerator magnet as well as insert coils to existing magnets. The technology is particularly suitable for $\mathrm{Nb}_{3} \mathrm{Sn}$ conductor as well as high temperature superconducting wires. In section II we give a short mathematical proof addressing the perfect dipole quality followed, in section III, with details on the magnet design and construction. In section IV we report test results and draw final conclusions.

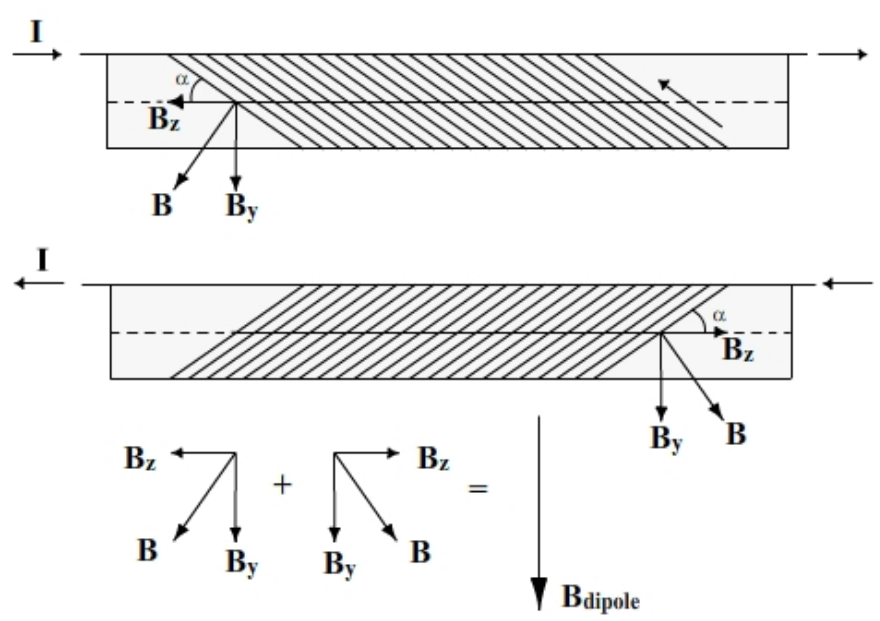

Fig. 1. Superposition of two alternating skewed solenoids generating a perfect dipole field.

\section{A. Mathematical Model}

A simplified mathematical proof is used to show that skewed windings on the surface of a cylindrical correspond to an axial current-density distribution that is $\cos \vartheta$ like, and therefore produce a "pure" dipole field. The current density flow lines that are distributed on the surface of the cylinder are elliptical (inclination angle $\alpha$ with respect to the cylinder axis) with flow lines coordinates expressed as (see Fig. 1-2) 
$x=R \cos \vartheta$

$y=R \sin \vartheta$

$z=y \cot \alpha=R \sin \vartheta \cot \alpha$

where $\mathrm{R}$ is the cylinder radius and $\vartheta$ is the azimuth angle. A normalized unit vector $\mathrm{S}$ along the current flow is:

$d S=R d \vartheta\left(\hat{e}_{\vartheta}+\cot \alpha \cos \vartheta \cdot \hat{e}_{z}\right)$

$d S /|d S|=\frac{\left(\hat{e}_{\vartheta}+\cot \alpha \cos \vartheta \cdot \hat{e}_{z}\right)}{\sqrt{\left(1+\cot ^{2} \alpha \cos ^{2} \vartheta\right)}}$.

The current density at any given location in $\mathrm{z}$ is a function of $\vartheta$ only, whereas $J=J_{0}(\vartheta)$ and its components are proportional to:

$J_{\vartheta} \sim \frac{J_{0}(\vartheta)}{\sqrt{\left(1+\cot ^{2} \alpha \cos ^{2} \vartheta\right)}} \quad J_{z} \sim \frac{J_{0}(\vartheta) \cot \alpha \cos \vartheta}{\sqrt{\left(1+\cot ^{2} \alpha \cos ^{2} \vartheta\right)}}$

Since the current density is divergence free, $\nabla \cdot J=0$, it follows that $J_{0}(\vartheta) \sim \sqrt{\left(1+\cot ^{2} \alpha \cos ^{2} \vartheta\right)}$.

Substituting the expression of $J_{0}(\vartheta)$ results in a constant azimuthal current density (solenoid field), and an axial current density proportional to $\cos \vartheta$ that corresponds to a pure dipole field, i.e.

$$
\begin{aligned}
& J_{\vartheta} \sim \text { const } \\
& J_{z} \sim \cos \vartheta
\end{aligned}
$$

If we superpose a second layer with a negative inclination angle $-\alpha$, the solenoidal field will vanish and the dipole field will double. By superposing a number of layer-pairs the dipole field can be farther enhanced.

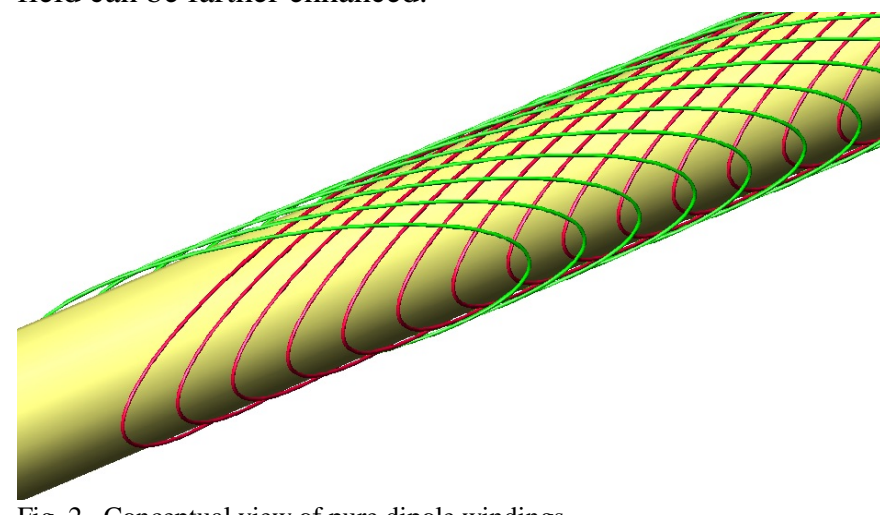

Fig. 2. Conceptual view of pure dipole windings.

\section{DESIGN AND CONSTRUCTION}

\section{A. Design}

The winding-mandrel was an aluminum tube (OD $25.4 \mathrm{~mm}$, ID $22.9 \mathrm{~mm}$, length $768 \mathrm{~mm}$ ) that also served as the magnet bore. The tube was wrapped with a $0.051 \mathrm{~mm}$ thick Kapton tape. A hollow nylon tube was snugly fitted within the bore to support two sets of pins used as magnet poles. The pins (stainless steel, $1 \mathrm{~mm}$ diameter and $6 \mathrm{~mm}$ long) were inserted into pin-holes machined into the aluminum tube and held firmly by the inner nylon tube. 147 pins were used along the "north" pole (top) and the same number along the "south" pole (bottom) (see Fig. 7). The spacing between pin was $1.76 \mathrm{~mm}$. Twenty additional pin holes were made along the "mid-plane" for pins assisting in the layer-to-layer transition.

The insulated $\mathrm{NbTi}$ wire $(0.6 \mathrm{~mm}$ diameter, $75 \mathrm{~m}$ long) was wound around the tube using the "top" and "bottom" pins to form a single layer. The angle $\alpha$ of each turn was 20 degrees with respect to the longitudinal axis (see Fig. 7) corresponding to an equivalent distance of 42 pins between "top" and "bottom" pins. Along the "mid-plane" the windings were made to nest-fit against each other. Before transitioning into a subsequent layer, Mylar inserts fillers were attached to support the end windings. Over each layer a wrapping of $0.025 \mathrm{~mm}$ thick Kapton tape was used as a precautionary layer to layer insulation. Transitioning from one layer to the next also required a reversal in the tilt angle $\alpha$ and the winding direction. A total of 4 layers were wound (Fig. 3). The effective overall magnet length, including its ends and straight section, was $350 \mathrm{~mm}(250 \mathrm{~mm}$ for ends and $100 \mathrm{~mm}$ for straight section).

The bare $\mathrm{NbTi}$ wire, insulated with a layer of thin Formvar coating ( $0.05 \mathrm{~mm}$ thick), had a copper to superconductor ratio of 1.37:1 and a current density in the superconductor of 1700 $\mathrm{A} / \mathrm{mm}^{2}$ at $4.2 \mathrm{~K}, 5 \mathrm{~T}$ (25 year old conductor). Without an iron yoke the expected short sample central field was $1.27 \mathrm{~T}$.

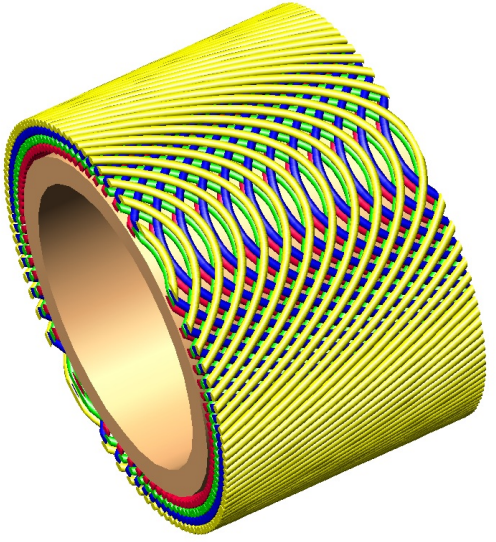

Fig. 3. Center cut of the 4 layer "cos-theta" dipole.

The cross section of the "cos-theta" like windings is shown in Fig. 4. The slight asymmetry in the wire positioning is a result of the winding pitch. An axial field scan at $78 \%$ of the bore radius is shown in Fig. 5. There is only a small field rise near the ends despite the lack of a return iron yoke.

The computed field harmonics along the magnet are shown in Fig. 6. The excellent field quality in the straight section (better than 0.1 units) was achieved without any optimization (no wedges) [9]. In addition, this end-type supports zero integrated normal harmonics over each individual end [10].

\section{B. Construction}

A rotating table and a tensioning unit were used during winding. A constant tension of $7 \mathrm{~N}$ was held during winding. Four NbTi layers were wound followed by a final wrap of Kevlar to provide an initial radial pre-compression to the coil. 
The complete coil was inserted into a custom-fit aluminum canister and vacuum impregnated. (Fig. 7 is a top view of layers 1 and 2).

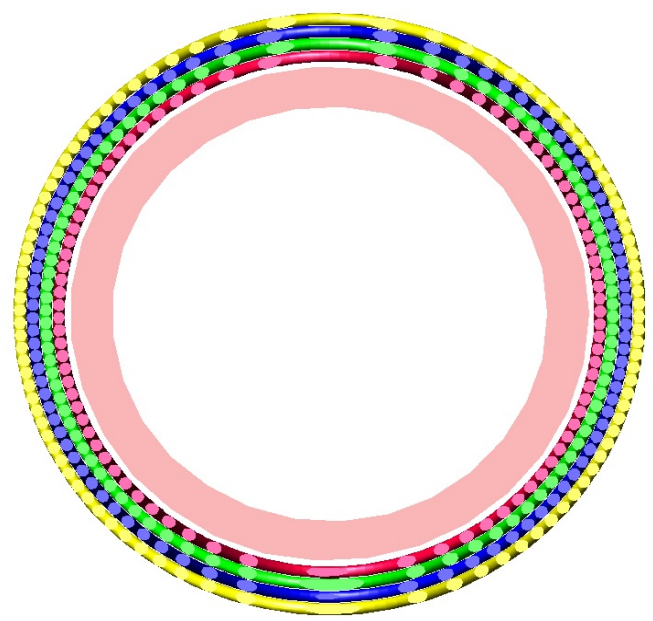

Fig. 4. Cross section of a 4 layers "cos-theta" dipole.

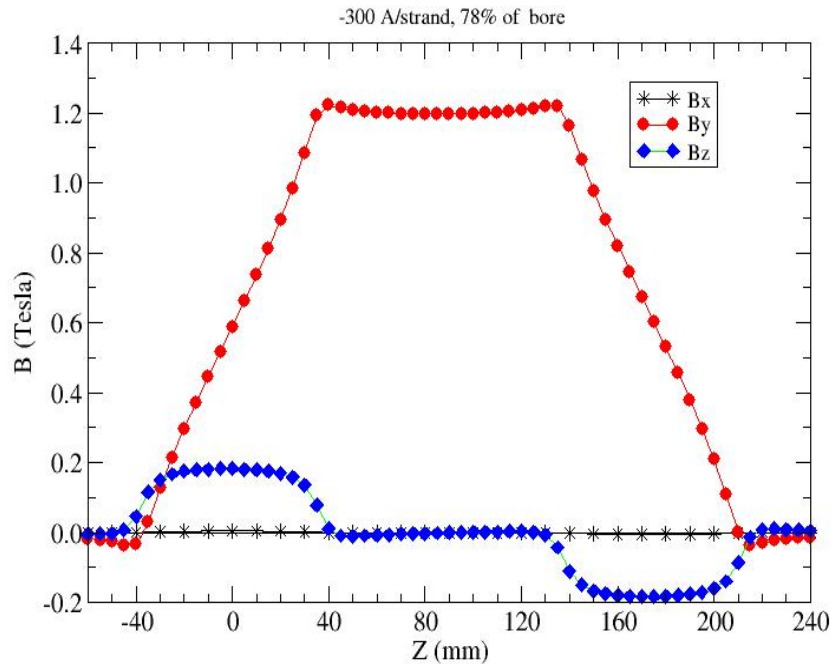

Fig. 5. Axial scan of field components at $X=0, Y=78 \% R$ ( $I=300 A)$.

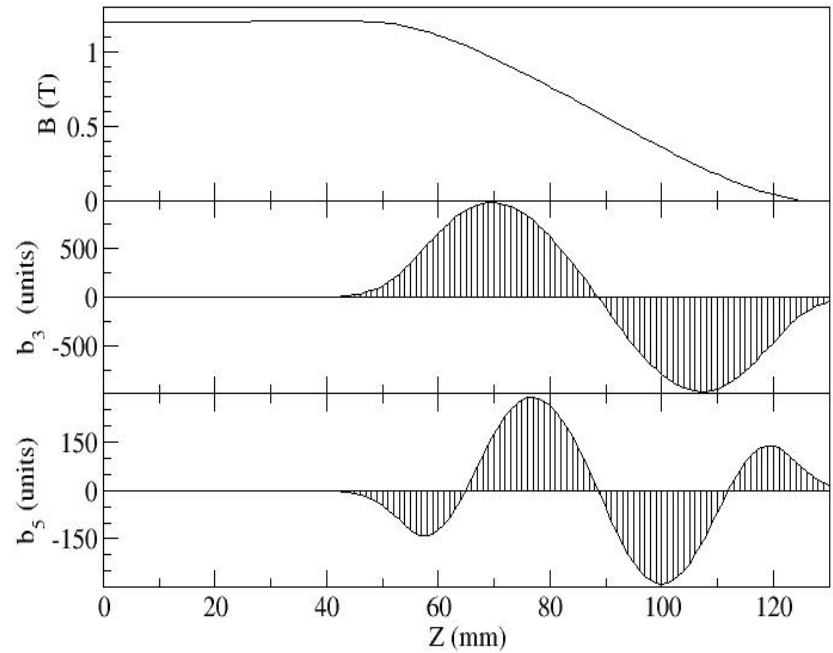

Fig. 6. Calculated dipole and harmonics through the magnet end.

The calculated "short sample" current and field are summarized in Table I for a case with and without an iron yoke.

Two post-mortem cuts were made following the test, one
TABLE I

SHORT SAMPLE CALCULATIONS

\begin{tabular}{ccc}
\hline \hline & Short Sample & Short Sample \\
& Current (A) & Field (T) \\
\hline No Iron, conductor & 318 & 1.377 \\
No Iron, bore & 318 & 1.27 \\
With Iron, bore & 300 & 1.535
\end{tabular}

Calculated short sample current and field at conductor and bore center

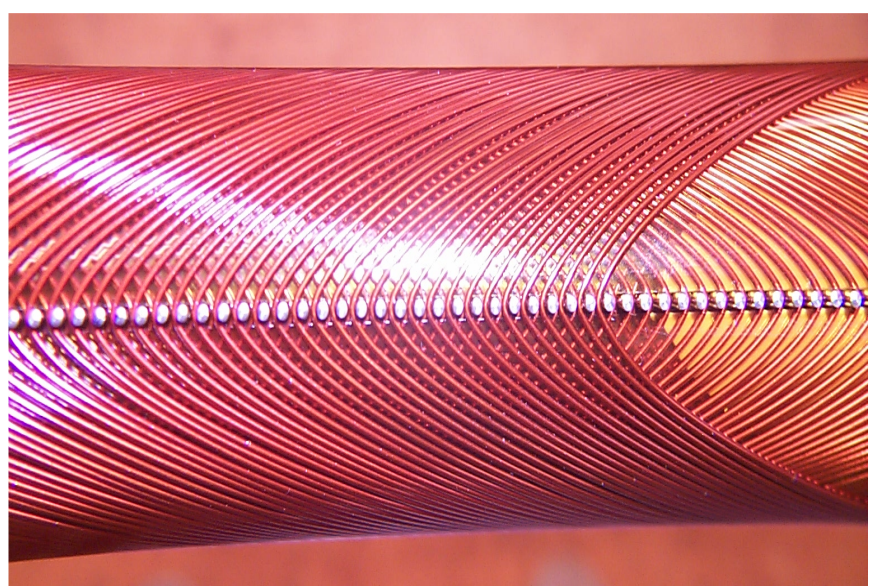

Fig. 7. Top view of two superposed layers (out of 4)

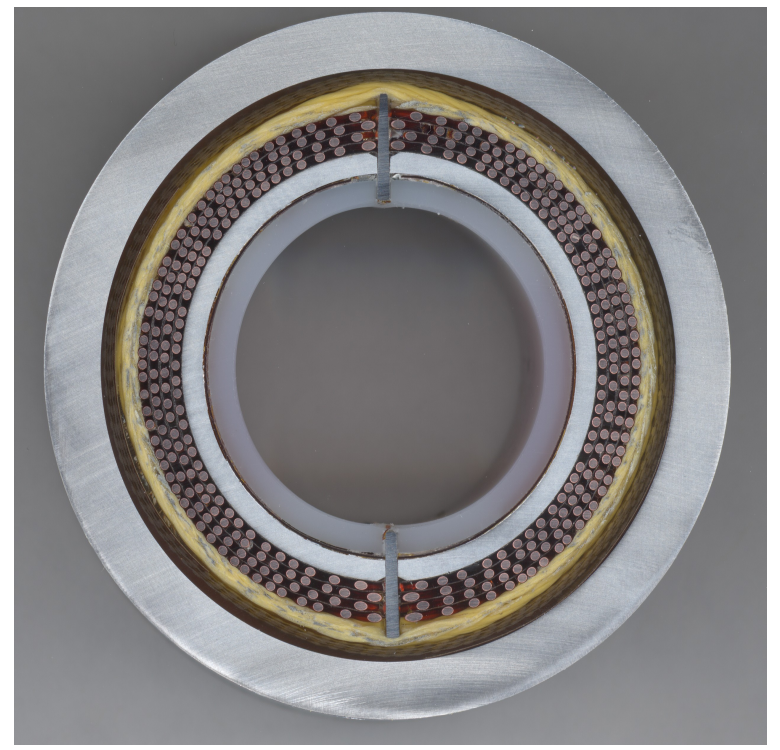

Fig. 8. Actual cross section of the dipole tested.

across the magnet and one along its axis (Fig. 8-9). The azimuthal winding distribution is not as perfect compared with the design shown in Fig. 4; however the general "cos-theta" arrangement is evident.

\section{RESULTS}

\section{A. Magnet test}

The magnet was tested twice with and without an iron yoke placed outside the aluminum canister. The field and current were measured in both cases and the load line obtained (Fig. 10). In both configurations the magnet started training around $50 \%$ of its expected short-sample and was still training when the test was terminated (Fig. 11). 


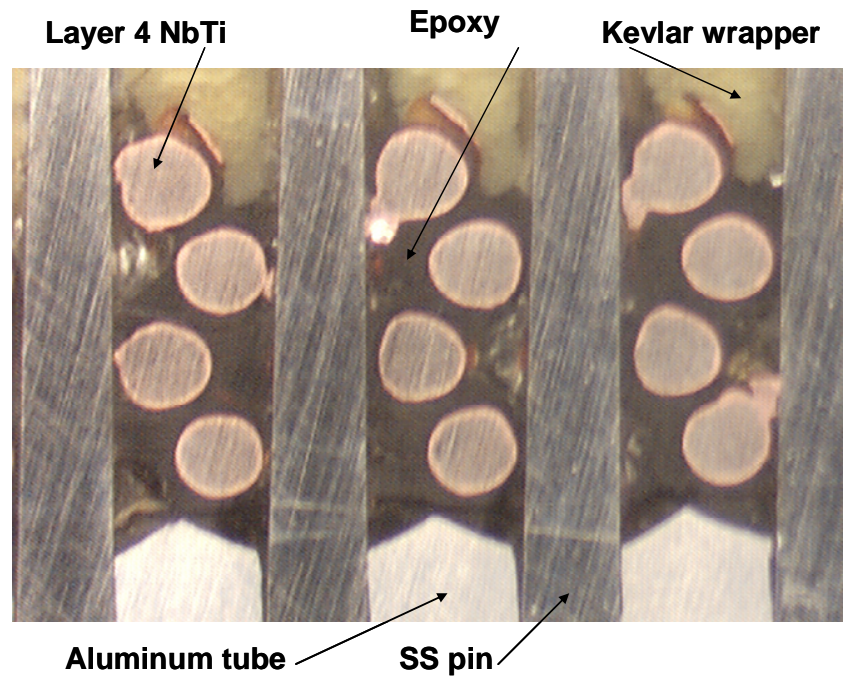

Fig. 9. Longitudinal cross-section along the pole.

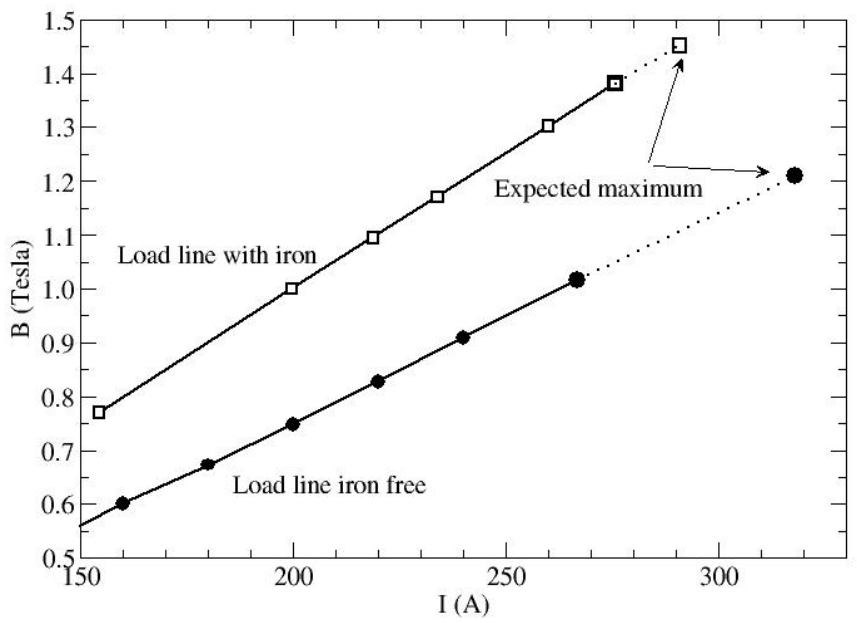

Fig. 10. Measured dipole field load lines

Without an iron yoke the magnet reached $80 \%$ of its short sample and with iron the magnet reached 90\% (Table II). A post mortem showed that the outer Kevlar wrapping was not wetted by the epoxy which can partially explain the excessive magnet training.

\section{CONCLUSION}

The use of superimposed tilted solenoid-windings is an efficient way to construct high quality accelerator magnets. The method promises to deliver a high field quality over a wide dynamic range, and it can be applied to any bore size. This configuration requires minimum handling, reduces end field effects, and provides a simple way for "grading" by varying the strand size in different layers. The design may be of interest as a "stand-alone" accelerator magnet (such as a superferric-magnet) or as inserts in high field magnets ( 16 T). The method may be quite adaptable to $\mathrm{Nb}_{3} \mathrm{Sn}$ and high $\mathrm{Tc}$ conductors. Extending this work to quadrupole magnets is underway and concerns on stress and magnet protection will be addressed in the future.
TABLE II

MEASURED VALUES

CURRENT (A)

FIELD (T)

$\begin{array}{ccc}\text { No Iron-Center } & 267 & 1.02 \\ \text { With Iron-Center } & 276 & 1.38\end{array}$

Measured results (the test was terminated before completing the training).

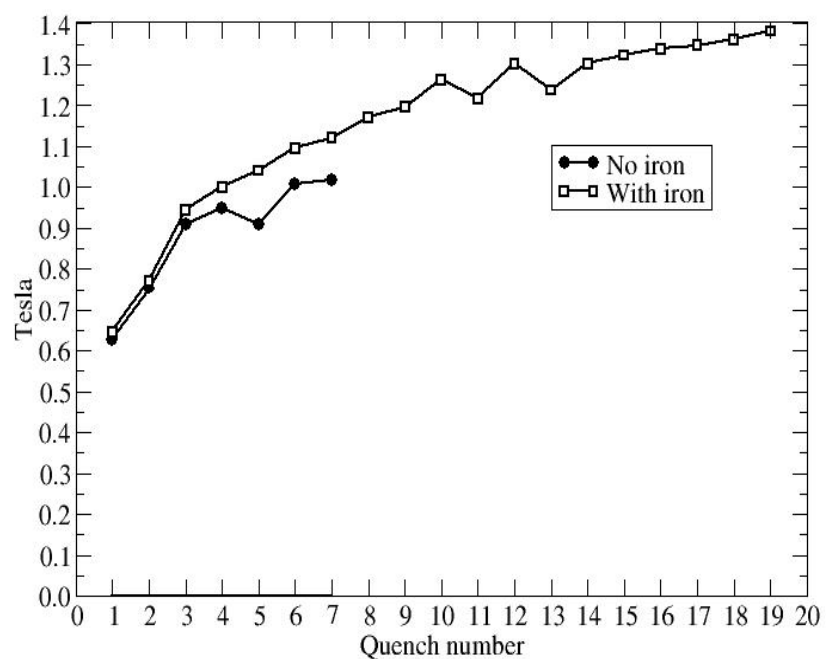

Fig. 11. Training curves with and without a return iron yoke. In both cases testing was stopped before the training process was finished.

\section{ACKNOWLEDGMENT}

We thank G. Ritchie for his excellent workmanship and interest during the magnet design and construction. We also thank H. Higley for his help during testing.

\section{REFERENCES}

[1] D.I. Meyer, and R. Flasck "A new configuration for a dipole magnet for use in high energy physics application", Nucl. Instr.and Methods 80, pp. 339-341, 1970.

[2] C.L. Goodzeit, M.J. Ball, and R.B. Meinke, "The Double-Helix dipolea novel approach to accelerator magnet design", IEEE Trans. Appl. Superconduct., Vol. 13, no. 2, pp. 1365-1368, June 2003.

[3] R.B. Meinke, et al., "Superconducting double-helix accelerator magnets" Proc. Particle. Acc. Conf., pp. 1996-1998, 2003.

[4] A.V. Gavrilin, et al., "New concepts in transverse field magnet design", IEEE Trans. Appl. Superconduct., Vol. 13, no. 2, pp. 1213-1216, June 2003.

[5] A.V. Gavrilin, et al., "Conceptual design of high transverse field magnets at NHMFL", IEEE Trans. Appl. Superconduct., Vol. 12, no. 1, pp. 465-469, March 2002.

[6] A. Devred, et al., "Overview and status of the next European dipole joint research activity”, Supercond. Sci. Technol. 19, pp 67-83, 2006.

[7] A. Devred, private communication.

[8] R.B. Meinke, C.L. Goodzeit and M.J. Ball, "Modulated Double-Helix quadrupole magnets", IEEE Trans. Appl. Superconduct., Vol. 13, no. 2, pp. 1369-1372, June 2003.

[9] L.J.Laslett, S.Caspi and M. Helm, "Configuration of coil ends for multipole magnets", Particle Accelerators Vol. 22, pp. 1-14, 1987.

[10] S. Caspi, M. Helm and L.J. Laslett, "The use of harmonics in 3D magnetic fields" IEEE Trans. On Magnetics, Vol. 30 pp. 2419-2422, July 1994 